\title{
New-onset postoperative seizures in patients with diffuse gliomas: a risk assessment analysis
}

\section{Lianwang Li}

Beijing Neurosurgical Institute, Capital Medical University, Beijing, 100070, China

\section{Guanzhang Li}

Beijing Neurosurgical Institute, Capital Medical University, Beijing, 100070, China

\section{Shengyu Fang}

Beijing Neurosurgical Institute, Capital Medical University, Beijing, 100070, China

\section{Kenan Zhang}

Beijing Neurosurgical Institute, Capital Medical University, Beijing, 100070, China

\section{Ruoyu Huang}

Beijing Neurosurgical Institute, Capital Medical University, Beijing, 100070, China

\section{Yinyan Wang}

Department of Neurosurgery, Beijing Tiantan Hospital, Capital Medical University, Beijing, 100070, China.

\section{Chuanbao Zhang}

Department of Neurosurgery, Beijing Tiantan Hospital, Capital Medical University, Beijing, 100070, China.

\section{Yiming Li}

Department of Neurosurgery, Beijing Tiantan Hospital, Capital Medical University, Beijing, 100070, China.

\section{Wei Zhang}

Department of Neurosurgery, Beijing Tiantan Hospital, Capital Medical University, Beijing, 100070, China.

\section{Zhong Zhang}

Department of Neurosurgery, Beijing Tiantan Hospital, Capital Medical University, Beijing, 100070, China.

\section{Qiang Jin}

Department of Neurosurgery, Beijing Tiantan Hospital, Capital Medical University, Beijing, 100070, China.

\section{Dabiao Zhou}

Department of Neurosurgery, Beijing Tiantan Hospital, Capital Medical University, Beijing, 100070, China.

\section{Xing Fan}

Beijing Neurosurgical Institute, Capital Medical University, Beijing, 100070, China

Tao Jiang ( $\square$ taojiang1964@163.com )

Beijing Neurosurgical Institute, Capital Medical University, Beijing, 100070, China

\section{Research Article}

Keywords: Diffuse glioma, New-onset postoperative seizures, Risk factors, IDH1 mutation

Posted Date: January 6th, 2021 
DOI: https://doi.org/10.21203/rs.3.rs-137298/v1

License: (c) (P) This work is licensed under a Creative Commons Attribution 4.0 International License. Read Full License 


\section{Abstract \\ Background}

Glioma-related epilepsy (GRE) is the most common presenting sign of patients with diffuse glioma. According to clinical experience, new-onset postoperative seizures can be observed even in patients without preoperative GRE. The current study mainly aimed to explore the risk factors of new-onset postoperative seizures in those patients. In addition, the prognostic value of new-onset postoperative seizures was also discussed.

\section{Methods}

Data of 313 patients without GRE were retrospectively reviewed. Chi-square test or Fisher's exact test were first performed to compare categorical variables between patients with new-onset postoperative seizures and those without. Subsequently, binary logistic regression analysis was conduct to further assess risk factors of newonset postoperative seizures. Kaplan-Meier and Cox analysis were used to investigate the prognostic value of new-onset postoperative seizures for progression-free survival (PFS) and overall survival (OS).

\section{Results}

Patients with low-grade tumors $(p=0.006)$, isocitrate dehydrogenase $1(\mathrm{IDH} 1)$ mutation $(p=0.040)$ or low Ki-67 expression $(p=0.005)$ showed a higher incidence of new-onset postoperative seizures. IDH1 mutation was identified as the only independent predictor for new-onset postoperative seizures (OR, 2.075; $95 \% \mathrm{Cl}, 1.051-$ 4.098; $p=0.035)$. Additionally, new-onset postoperative seizure occurrence was demonstrated as an independent predicter of prolonged OS $(\mathrm{OR}, 0.574 ; 95 \% \mathrm{Cl}, 0.335-0.983 ; \mathrm{p}=0.043)$.

\section{Conclusions}

IDH1 mutation is an independent predictor for new-onset postoperative seizures in patients without preoperative GRE. Moreover, new-onset postoperative seizures can independently predict prolonged OS in those patients. The results of the current study can contribute to improving the individualized management of diffuse glioma.

\section{Background}

Glioma-related epilepsy (GRE) is a common clinical symptom of diffuse gliomas. It can be observed in $65-90 \%$ of cases with diffuse low-grade gliomas (WHO grade 2) and $40-64 \%$ of cases with diffuse high-grade gliomas (WHO grades 3 and 4), with a clinical presentation varied from focal to tonic-clonic seizures ${ }^{1}$. GRE can significantly decrease the patients' quality of life, and bring heavy financial and psychological burdens to them and their families ${ }^{2}$.

For a long time, the sole purpose of glioma treatment has been to prolong survival. In recent decades, due to the increasing concern of the patients' quality of life, seizure control has been considered as a secondary goal for glioma treatment ${ }^{3}$. Currently, the optimal treatment strategy for GRE is a combination of antiepileptic drugs 
(AEDs) and antitumor treatments, including surgical resection and adjuvant radiotherapy and/or chemotherapy

${ }^{1}$. However, despite the aforementioned treatment strategy, seizure control is still not achieved in over $20 \%$ of patients ${ }^{4}$.

Factors associated with postoperative seizure control have been investigated in numerous studies ${ }^{5,6}$. In 2018 , we performed a systemic review and meta-analysis basing on relevant studies, and identified that younger age, clinical presentation of focal seizures, a prolonged history of seizures prior to surgery and non-gross total resection (non-GTR) had negative impacts on postoperative seizure control ${ }^{7}$. For patients with the above risk factors, aggressive AEDs therapy is potentially helpful for improving seizure control. In addition, several surgical modalities can be applied to achieve potential better seizure outcome in patients with preoperative GRE. For instance, electroencephalogram (EEG) and/or intra-operative electrocorticography can be applied to facilitate the identification of the epileptogenic zone (EZ), and extended resection and/or multiple subpial transection can be used to remove the potential EZ ${ }^{8}$.

It's worth noting that postoperative seizures can occur even in some patients without preoperative GRE ${ }^{9}$. This subset of patients should be of particular concern. The absence of preoperative seizure history prevents them from receiving antiepileptic surgical procedures and aggressive AED therapy, from which they could have benefit a lot. Accordingly, characterizing this subset of patients is of great significance for the individualized management of diffuse glioma. In the current study, we aimed mainly to explore the risk factors of new-onset postoperative seizures in patients without preoperative GRE, moreover, the association between new-onset postoperative seizures and patient survival was also investigated.

\section{Methods}

\subsection{Study population}

Data of patients who underwent surgical resection at Beijing Tiantan Hospital between January 2006 and December 2018 were retrospectively reviewed. The inclusion criteria were as follows: 1) newly diagnosed and histologically confirmed supratentorial diffuse glioma; 2) no history of preoperative seizures; 3 ) age between 18 and 75 years old. The exclusion criteria were as follows: 1) history of biopsy, radiotherapy and chemotherapy prior to operation; 2) previous malignancy or other concomitant malignant disease; 3) multifocal tumors. Ultimately, a total of 313 patients were enrolled. Demographic, clinical and follow-up data were collected from the Chinese Glioma Genome Atlas (CGGA) database (http://www.cgga.org.cn/). The study was approved by the Ethics Committee of Beijing Tiantan Hospital, and written informed consent was obtained from all the patients or their legal guardians.

\subsection{Histopathological diagnosis and molecular markers}

Tumor samples obtained from surgical resection were immediately snap-frozen in liquid nitrogen. Subsequently, hematoxylin and eosin-stained sections were prepared to assess the percentage of tumor cells, samples with over $80 \%$ tumor cells were selected for further analysis. The histopathological diagnosis was made independently by two experienced neuropathologists. Any inconsistent judgments would be resolved by a third expert. As for molecular markers, isocitrate dehydrogenase 1 (IDH1) mutation was detected by pyrosequencing, while p53 and Ki-67 expression were assessed via immunohistochemistry. 


\subsection{Evaluation of the extent of resection}

Pre- and postoperative magnetic resonance images (within 72 hours after surgery) were reviewed. The extent of resection (EOR) was stratified as gross total resection (GTR) and non-GTR. For non-enhancing tumors, GTR was defined as no residual high-signal area on postoperative T2-weighted images. For enhancing tumors, GTR was defined as no residual high-signal area on postoperative contrast-enhanced T1-weighted images. The EOR was evaluated independently by two experienced neurosurgeons who were blinded to the clinical outcomes. Cases with inconsistent results would be reviewed by a third neurosurgeon for final decision.

\subsection{Follow-up}

All patients received prophylactic AEDs (valproate or levetiracetam) after surgery. Follow-up data were collected by scheduled clinical visits or telephone interviews. The collected information included postoperative seizure occurrence, adjuvant therapy (radiotherapy and/or chemotherapy), AEDs regimen, progression-free survival (PFS) and overall survival (OS). PFS was defined as the time from surgery to radiographic progression (identified according to the Response Assessment in Neuro-Oncology criteria), and OS was defined as the time from surgery until death.

\subsection{Statistical analysis}

SPSS software (version 16.0, SPSS Inc., Chicago, IL, USA) were used for data management and statistical analysis. Chi-square test or Fisher's exact test were used for comparison of categorical variables between patients with new-onset postoperative seizures and those without. Binary logistic regression analysis with backward stepwise selection was performed to identify independent risk factors associated with new-onset postoperative seizures. For survival analysis, Kaplan-Meier method (log-rank test) was applied to investigate the correlation of new-onset postoperative seizures with PFS and OS. The R project for statistical computing (version 4.0.0, available at www.r-project.org) was used for graphic plotting (survival curves and nomograms) with packages including "survival", "ggplot2" and "rms". At last, backward stepwise Cox regression analysis was used to further evaluate the prognostic value of new-onset postoperative seizures. A p value $<0.05$ was considered statistically significant. Odds ratio (OR) and $95 \%$ confidence interval $(\mathrm{Cl})$ were used to evaluate the correlations and measure relevant results.

\section{Results}

\subsection{Patient characteristics}

Among the enrolled 313 patients without preoperative GRE, ninety-six patients experienced new-onset postoperative seizures, while the other 217 did not. Clinical characteristics of enrolled patients are summarized in Table 1. Low-grade gliomas $(p=0.006)$, IDH1 mutation $(p=0.040)$ and low Ki-67 expression (defined as a Ki67 index $<10 \%, p=0.005$ ) were identified to be associated with a higher incidence of new-onset postoperative seizures. No significant difference was detected in age, sex, tumor side, temporal lobe involvement, preoperative functional deficits, EOR, p53 expression, postoperative adjuvant therapy between patients with new-onset postoperative seizures and those without. 
Table 1

Clinical characteristics of enrolled patients

\begin{tabular}{|c|c|c|c|c|}
\hline Variables & Total & New-onset seizures & No seizures & $P$ value \\
\hline No. of patients & 313 & 96 & 217 & \\
\hline Sex & & & & 0.625 \\
\hline Male & 176 & 52 & 124 & \\
\hline Female & 137 & 44 & 93 & \\
\hline \multicolumn{5}{|l|}{ Age } \\
\hline$<45$ yrs & 163 & 54 & 109 & 0.326 \\
\hline$\geq 45$ yrs & 150 & 42 & 108 & \\
\hline Side & & & & 0.835 \\
\hline Left & 136 & 41 & 95 & \\
\hline Right & 155 & 47 & 108 & \\
\hline Bilateral & 22 & 8 & 14 & \\
\hline Location & & & & 0.231 \\
\hline Temporal lobe & 131 & 45 & 86 & \\
\hline Others & 182 & 51 & 131 & \\
\hline Functional deficits & & & & 0.097 \\
\hline Yes & 126 & 32 & 94 & \\
\hline No & 187 & 64 & 123 & \\
\hline EOR & & & & 0.777 \\
\hline GTR & 157 & 47 & 110 & \\
\hline Non-GTR & 156 & 49 & 107 & \\
\hline WHO grade & & & & $0.006^{*}$ \\
\hline Low-grade & 149 & 57 & 92 & \\
\hline High-grade & 164 & 39 & 125 & \\
\hline IDH1 mutation & & & & $0.040^{*}$ \\
\hline Yes & 165 & 57 & 108 & \\
\hline No & 109 & 25 & 84 & \\
\hline
\end{tabular}

EOR, extent of resection; GTR, gross total resection; IDH1, isocitrate dehydrogenase 1

${ }^{*} \mathrm{P}<0.05$ was considered as statistically significant. 


\begin{tabular}{|lcccc|}
\hline Variables & Total & New-onset seizures & No seizures & $P$ value \\
\hline p53 expression & & & & 0.293 \\
\hline Over-expressed & 150 & 40 & 110 & \\
\hline Not & 107 & 35 & 72 & $0.005^{*}$ \\
\hline Ki-67 expression & & & & \\
\hline Low & 116 & 46 & 70 & 0.260 \\
\hline High & 108 & 24 & 84 & \\
\hline Combined radiochemotherapy & & & & \\
\hline Yes & 151 & 55 & 96 & \\
\hline No & 136 & 41 & & \\
\hline EOR, extent of resection; GTR, gross total resection; IDH1, isocitrate dehydrogenase 1 & \\
\hline *P $<0.05$ was considered as statistically significant. & & \\
\hline
\end{tabular}

\subsection{Risk factors for new-onset postoperative seizures}

Multivariate binary logistic regression analysis was applied to determine risk factors for new-onset postoperative seizures. All relevant ordinal categorical variables were incorporated into the regression model. Due to incomplete data, only 190 patients were analyzed. Only IDH1 mutation was identified as an independent predictor for new-onset postoperative seizures $(\mathrm{OR}, 2.075 ; 95 \% \mathrm{Cl}, 1.051-4.098 ; \mathrm{p}=0.035)$. A nomogram for new-onset postoperative seizure occurrence is shown in Fig. 1.

\subsection{Prognostic value of new-onset postoperative seizure in diffuse gliomas}

Among the enrolled 313 patients, 40 patients were lost to follow-up, the follow-up rate was $87.22 \%$. The mean follow-up time was 55.33 months (range 5.73-165.43 months). Kaplan-Meier analysis was performed to investigate the prognostic value of new-onset postoperative seizures. The result showed that patients with postoperative new-onset postoperative seizures experienced longer OS compared with those without $(p=0.025$, log-rank test, Fig. 2A). In contrast, no significant difference was detected in PFS between the two groups ( $p=$ 0.174 , log-rank test, Fig. 2B). Subsequently, univariate and multivariate Cox proportional hazard regression analysis was performed to further explore the prognostic value of new-onset postoperative seizures. 171 patients with available complete data were incorporated into the model. The results are shown in Table 2. Newonset postoperative seizures was identified as an independent predicter of prolonged OS $(\mathrm{OR}, 0.574 ; 95 \% \mathrm{Cl}$, $0.335-0.983 ; p=0.043)$. In addition to new-onset postoperative seizures, younger age $(p=0.002), G T R(p<$ $0.001)$, low-grade $(p<0.001)$, IDH1 mutation $(p=0.007)$ and low p53 expression $(p=0.005)$ were shown to be independently associated with prolonged OS. A prognostic nomogram for OS is presented in Fig. 3. As for PFS, younger age $(p=0.009)$, GTR $(p<0.001)$, low-grade $(p=0.001)$, IDH mutation $(p=0.004)$ and low $p 53$ expression $(p=0.001)$ were identified as independent positive predictors. 
Table 2

Univariate and multivariate COX analysis of risk factors associated with survival in patients with diffuse gliomas

\begin{tabular}{|c|c|c|c|c|c|c|c|c|}
\hline \multirow[t]{3}{*}{ Variable } & \multicolumn{4}{|l|}{ OS } & \multicolumn{4}{|l|}{ PFS } \\
\hline & \multirow{2}{*}{$\begin{array}{l}\text { Univariable } \\
P\end{array}$} & \multicolumn{3}{|c|}{ Multivariable } & \multirow{2}{*}{$\begin{array}{l}\text { Univariable } \\
P\end{array}$} & \multicolumn{3}{|c|}{ Multivariable } \\
\hline & & HR & $95 \% \mathrm{Cl}$ & $P$ & & HR & $95 \% \mathrm{Cl}$ & $P$ \\
\hline Male & 0.934 & & & & 0.963 & & & \\
\hline Age $\geq 45$ yrs & $<0.001^{\star}$ & 2.266 & $\begin{array}{l}1.348- \\
3.810\end{array}$ & 0.002 & $<0.001 *$ & 1.935 & $\begin{array}{l}1.178- \\
3.178\end{array}$ & $0.009 *$ \\
\hline Temporal lobe & 0.205 & & & & 0.094 & & & \\
\hline Functional deficits & $0.004^{\star}$ & & & & $0.004^{\star}$ & & & \\
\hline GTR & $<0.001^{\star}$ & 0.159 & $\begin{array}{l}0.090- \\
0.280\end{array}$ & <.001* & $<0.001^{\star}$ & 0.218 & $\begin{array}{l}0.132- \\
0.361\end{array}$ & 0.001* \\
\hline High WHO grade & $<0.001^{\star}$ & 2.616 & $\begin{array}{l}1.541- \\
4.442\end{array}$ & <. $0.001 *$ & $<0.001^{\star}$ & 2.300 & $\begin{array}{l}1.420- \\
3.725\end{array}$ & $0.001 *$ \\
\hline IDH1 Mutation & $<0.001^{\star}$ & 0.482 & $\begin{array}{l}0.283- \\
0.823\end{array}$ & $0.007 *$ & $<0.001^{\star}$ & 0.472 & $\begin{array}{l}0.283- \\
0.786\end{array}$ & $0.004 *$ \\
\hline $\begin{array}{l}\text { p53 over- } \\
\text { expression }\end{array}$ & $0.016^{\star}$ & 2.209 & $\begin{array}{l}1.272- \\
3.838\end{array}$ & $0.005^{\star}$ & $0.006^{\star}$ & 2.338 & $\begin{array}{l}1.397- \\
3.913\end{array}$ & $0.001 *$ \\
\hline $\begin{array}{l}\text { Ki-67 high } \\
\text { expression }\end{array}$ & $0.002^{\star}$ & & & & $0.001 *$ & & & \\
\hline New-onset seizures & $0.026 *$ & 0.574 & $\begin{array}{l}0.335- \\
0.983\end{array}$ & $0.043^{\star}$ & 0.175 & & & \\
\hline $\begin{array}{l}\text { Combined } \\
\text { radiochemotherapy }\end{array}$ & $0.002^{\star}$ & & & & $<0.001^{\star}$ & & & \\
\hline \multicolumn{9}{|c|}{$\begin{array}{l}\text { OS, overall survival; PFS, progression-free survival; GTR, gross total resection; IDH1, isocitrate } \\
\text { dehydrogenase 1; OR, odds ratio; Cl, confidence interval }\end{array}$} \\
\hline
\end{tabular}

\section{Discussion}

In terms of postoperative management for diffuse glioma, there are many controversial issues that need to be clarified. The use of prophylactic AEDs in patients without preoperative GRE is definitely one of them ${ }^{1}$. There is a plenty of evidences to prove that AED prophylaxis do not significantly reduce the incidence of postoperative seizures in brain tumor patients without preoperative seizures ${ }^{10,11}$. Accordingly, Many experts believe that routine prophylactic AED use has no benefit for this group of patients ${ }^{12}$. However, in our opinion, AED prophylaxis is not meaningless for patients without preoperative GRE, as new-onset postoperative seizures can indeed be observed in some of them. A more plausible explanation is that for one subset of patients, routine AED prophylaxis is unnecessary or at least sufficient for postoperative seizure control; while for the other subset 
of patients, routine AED prophylaxis is insufficient and should be more aggressive. In consequence, the identification of markers for distinguishing between the two subsets is of great importance.

In the current study, we explored the risk factors of new-onset postoperative seizures in patients without preoperative GRE, and identified that IDH1 mutation was the only independent predictor for new-onset postoperative seizures by multivariate analysis. The result suggested that for patients with IDH1 mutant gliomas, more aggressive AED regimen should be adopted even if they did not have preoperative GRE. IDH1 mutation is considered as an early event in glioma-genesis, and has gained major importance in the 2016 WHO classification of diffuse gliomas ${ }^{13}$. In addition, the close correlation of IDH1 mutation with preoperative GRE has already been confirmed ${ }^{14}$. The accumulation of D-2-hydroxyglutarate (D2HG), which is structurally similar to the major excitatory neurotransmitter glutamate, is supposed to be a major mechanism behind such correlation. As an important metabolic enzyme, IDH1 can catalyze the oxidative decarboxylation of isocitrate to a-ketoglutarate. While mutant IDH1 can reduce a-ketoglutarate to D2HG, which results in the accumulation of D2HG in tumor microenvironment. The accumulation of D2HG can lead to overexcitation of neurons and thus induce epilepsy 15,16 . This mechanism can also explain the association between IDH1 mutation and new-onset postoperative seizures. Generally, we evaluate the EOR basing on tumor boundary seen on neuroimaging. However, a radiologically proven GTR does not mean that all tumor cells have been removed, and the presence of glioma cells outside the radiological tumor borders has been demonstrated ${ }^{17}$. The remaining IDH1 mutant cells can still affect the surrounding microenvironment, and lead to new-onset seizures. Overall, in order to reduce the incidence of postoperative GRE, a GTR basing on the "epileptogenic boundary", which is actually the histological boundary, can be more effective.

Another potential mechanism behind the correlation of IDH1 mutation with new-onset postoperative seizures is related to seizure spread network. It is reported that patients with IDH1 mutant gliomas have higher global

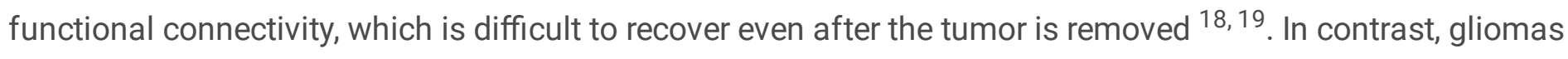
carrying wild-type IDH1 are often more aggressive and thus more likely to cause destruction of brain networks, which may add barriers to the spread of epileptic discharges ${ }^{20}$.

We also found that WHO grade and Ki-67 expression were negatively associated with new-onset postoperative seizures. It has been widely accepted that patients with low-grade gliomas have a higher incidence of preoperative GRE ${ }^{1}$. In the current study, we identified that low-grade glioma patients without preoperative GRE also have a higher incidence of new-onset postoperative seizures. IDH1 mutant status may be one of the decisive factors behind such relationship, because IDH1 mutation is frequently observed in diffuse gliomas but rarely seen in primary glioblastomas $(\mathrm{WHO} \text { grade } \nabla)^{20}$. Maybe that was also why WHO grade showed no statistical significance in regression analysis. As for $\mathrm{Ki}-67$, it is an effective biomarker to determine the proliferative activity of tumors ${ }^{21}$. As mentioned above, tumors with stronger proliferation ability are more likely to cause damage to brain networks, and impede the spread of epileptic discharges. Therefore, it's not difficult to understand the association between low Ki-67 expression and new-onset postoperative seizures.

The prognostic value of new-onset postoperative seizures was also investigated. In 2018, we performed a metaanalysis to determine the association between preoperative GRE and clinical outcomes, and identified that GRE at presentation is significantly correlated with prolonged OS in patients with diffuse glioma ${ }^{14}$. Consistent with this previous study, here we also demonstrated that new-onset postoperative seizures could predict longer OS in 
patients without preoperative GRE. Given the correlation of new-onset postoperative seizures with the above proven positive prognostic factors, like lower WHO grade and IDH1 mutation, such result was predictable ${ }^{22,23}$. However, early postoperative seizures do not seem to predict a good prognosis. Dewan et al. reported that glioma patients with early postoperative seizures have a significant shorter median survival ( 3 months) than those without (15.6 months) ${ }^{24}$. The worse prognosis of patients with early postoperative seizures may due to the hemorrhage and severe intracranial hypertension following epilepsy ${ }^{25}$. For patients with early postoperative seizures, corresponding active examinations and treatments should be proceed in a timely manner.

The study has several limitations. For instance, as a proportion of patients were followed up by telephone interviews, EEG was not applied for the definite diagnosis of seizures in those patients. Moreover, the study was limited by its retrospective nature, the results need to be verified in a prospective set of patients. Efforts to further explore risk factors of new-onset postoperative seizures, and reduce the incidence of postoperative seizures through patient-tailored antiepileptic therapies should remain the priorities of future studies.

\section{Conclusions}

The current study identified that IDH1 mutation was an independent risk factor for new-onset postoperative seizures in patients without preoperative GRE. Moreover, new-onset postoperative seizure occurrence is demonstrated as an independent predictor of prolonged OS in those patients. To the best of our knowledge, this is the first study to investigate the above topics in patients with diffuse glioma. The results can provide new evidences for improving the management of diffuse glioma, and benefit relevant patients from the application of tailored antiepileptic therapies.

\section{Abbreviations}

GRE, glioma-related epilepsy; PFS, progression-free survival; OS, overall survival; IDH1, isocitrate dehydrogenase 1; AEDs, antiepileptic drugs; EOR, extent of resection; GTR, gross total resection; EEG, electroencephalogram; EZ, epileptogenic zone; CGGA, Chinese Glioma Genome Atlas.

\section{Declarations}

Ethics approval and consent to participate: The study was approved by the Ethics Committee of Beijing Tiantan Hospital, and written informed consent was obtained from all the patients or their legal guardians. All procedures have been carried out ethically in accordance with the World Medical Association Declaration of Helsinki.

Consent for publication: Not applicable. No identified information about participants was included in the article.

Availability of data and material: The datasets used and/or analyzed during the current study are available from the corresponding author on reasonable request.

Conflicts of interest: The authors declare that they have no competing interests.

Funding: This work was supported by the National Natural Science Foundation of China [grant number, 82001777, Xing Fan]; the Public welfare development and reform pilot project of Beijing Medical Research 
Institute [grant number, JYY 2019-5, Tao Jiang]; the CAMS Innovation Fund for Medical Sciences [grant number, 2019-I2M-5-021, Tao Jiang] and the Beijing Nova Program [grant number, Z181100006218064, Yinyan Wang].

\section{Authors' contributions:}

Study concept and design: Lianwang Li and Xing Fan;

Data acquisition and analysis: Lianwang Li, Guanzhang Li, Kenan Zhang and Shengyu Fang;

Formal analysis and investigation: Lianwang Li and Ruoyu Huang;

Writing - Original Draft preparation: Lianwang Li;

Writing - review and editing: Xing Fan and Yiming Li;

Funding acquisition: Yinyan Wang and Tao Jiang;

Resources: Chuanbao Zhang, Wei Zhang, Zhong Zhang, Qiang Jin and Dabiao Zhou;

Supervision: Xing Fan and Tao Jiang.

Acknowledgements: Not applicable.

\section{References}

1. Liang S, Fan X, Zhao M, et al. Clinical practice guidelines for the diagnosis and treatment of adult diffuse glioma-related epilepsy. Cancer Med 2019;8:4527-4535.

2. Shin JY, Kizilbash SH, Robinson SI, et al. Seizures in patients with primary brain tumors: what is their psychosocial impact? J Neurooncol 2016;128:285-291.

3. Avila EK, Chamberlain M, Schiff D, et al. Seizure control as a new metric in assessing efficacy of tumor treatment in low-grade glioma trials. Neuro Oncol 2017;19:12-21.

4. Goldstein ED, Feyissa AM. Brain tumor related-epilepsy. Neurol Neurochir Pol 2018;52:436-447.

5. Yang P, Liang T, Zhang C, et al. Clinicopathological factors predictive of postoperative seizures in patients with gliomas. Seizure 2016;35:93-99.

6. Xu DS, Awad AW, Mehalechko C, et al. An extent of resection threshold for seizure freedom in patients with low-grade gliomas. J Neurosurg 2018;128:1084-1090.

7. Shan X, Fan X, Liu X, Zhao Z, Wang Y, Jiang T. Clinical characteristics associated with postoperative seizure control in adult low-grade gliomas: a systematic review and meta-analysis. Neuro Oncol 2018;20:324-331.

8. Yao PS, Zheng SF, Wang F, Kang DZ, Lin YX. Surgery guided with intraoperative electrocorticography in patients with low-grade glioma and refractory seizures. J Neurosurg 2018;128:840-845.

9. Oushy S, Sillau SH, Ney DE, et al. New-onset seizure during and after brain tumor excision: a risk assessment analysis. J Neurosurg 2018;128:1713-1718.

10. Sirven JI, Wingerchuk DM, Drazkowski JF, Lyons MK, Zimmerman RS. Seizure prophylaxis in patients with brain tumors: a meta-analysis. Mayo Clin Proc 2004;79:1489-1494. 
11. Delgado-Lopez PD, Martin-Alonso J. Prophylactic anticonvulsant therapy in high-grade glioma: $A$ systematic review and meta-analysis of longitudinal studies. Neurocirugia (Astur) 2020.

12. Glantz MJ, Cole BF, Forsyth PA, et al. Practice parameter: anticonvulsant prophylaxis in patients with newly diagnosed brain tumors. Report of the Quality Standards Subcommittee of the American Academy of Neurology. Neurology 2000;54:1886-1893.

13. Louis DN, Perry A, Reifenberger G, et al. The 2016 World Health Organization Classification of Tumors of the Central Nervous System: a summary. Acta Neuropathol 2016;131:803-820.

14. Fan X, Li Y, Shan X, et al. Seizures at presentation are correlated with better survival outcomes in adult diffuse glioma: A systematic review and meta-analysis. Seizure 2018;59:16-23.

15. Chen $\mathrm{H}$, Judkins $\mathrm{J}$, Thomas $\mathrm{C}$, et al. Mutant IDH1 and seizures in patients with glioma. Neurology 2017;88:1805-1813.

16. Wychowski T, Kapur J. Isocitrate dehydrogenase mutations: A biomarker for glioma-related excitability and seizures. Neurology 2017;88:1782-1783.

17. Zetterling M, Roodakker KR, Berntsson SG, et al. Extension of diffuse low-grade gliomas beyond radiological borders as shown by the coregistration of histopathological and magnetic resonance imaging data. J Neurosurg 2016;125:1155-1166.

18. Kesler SR, Noll K, Cahill DP, Rao G, Wefel JS. The effect of IDH1 mutation on the structural connectome in malignant astrocytoma. J Neurooncol 2017;131:565-574.

19. Derks J, Kulik S, Wesseling P, et al. Understanding cognitive functioning in glioma patients: The relevance of IDH-mutation status and functional connectivity. Brain Behav 2019;9:e01204.

20. Cancer Genome Atlas Research N, Brat DJ, Verhaak RG, et al. Comprehensive, Integrative Genomic Analysis of Diffuse Lower-Grade Gliomas. N Engl J Med 2015;372:2481-2498.

21. Fisher BJ, Naumova E, Leighton CC, et al. Ki-67: a prognostic factor for low-grade glioma? Int J Radiat Oncol Biol Phys 2002;52:996-1001.

22. Sanson M, Marie Y, Paris S, et al. Isocitrate dehydrogenase 1 codon 132 mutation is an important prognostic biomarker in gliomas. J Clin Oncol 2009;27:4150-4154.

23. Ostrom QT, Bauchet L, Davis FG, et al. The epidemiology of glioma in adults: a "state of the science" review. Neuro Oncol 2014;16:896-913.

24. Dewan MC, White-Dzuro GA, Brinson PR, Thompson RC, Chambless LB. Perioperative seizure in patients with glioma is associated with longer hospitalization, higher readmission, and decreased overall survival. $J$ Neurosurg 2016;125:1033-1041.

25. Klimek M, Dammers R. Antiepileptic drug therapy in the perioperative course of neurosurgical patients. Curr Opin Anaesthesiol 2010;23:564-567.

\section{Figures}


Points

Sex

Age

Location

Functional deficits

EOR

WHO grade

IDH1 mutation

p53 expression

Ki-67 expression

Combined radiochemotherapy

Total Points

New-onset seizures
0

2

Female

$$
\text { Male }
$$

$\geq 45 \mathrm{yrs}$

$<45 \mathrm{yrs}$

$\begin{array}{ll}\geq 45 \text { yrs } & \text { Temporal lobe } \\ \text { Others } & \text { No }\end{array}$

Yes

GTR

High-grade

Low-grade

Non-GTR

(

No

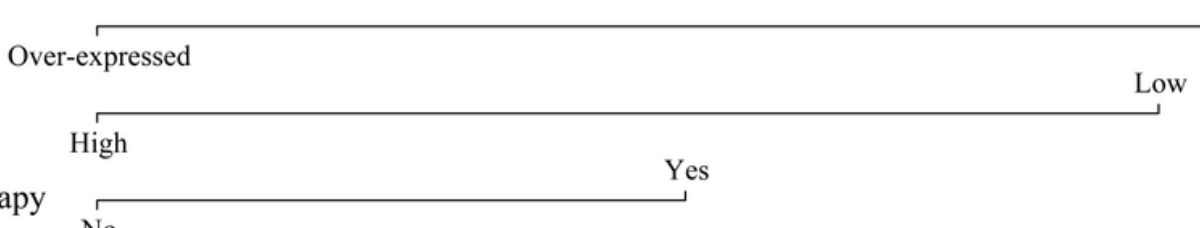

No$$
0
$$

0

$$
0.1
$$

10
30
Yes

Not

8

10

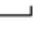

\section{ow}

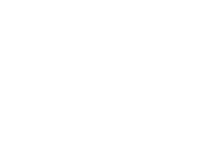

\section{Figure 1}

Predictive nomogram for new-onset postoperative seizures. The nomogram showed risk factors of new-onset seizure in patients with diffuse gliomas. EOR, extent of resection; WHO, World Health Organization; 
A

Strata $\mp$ No seizures + New-onset seizures

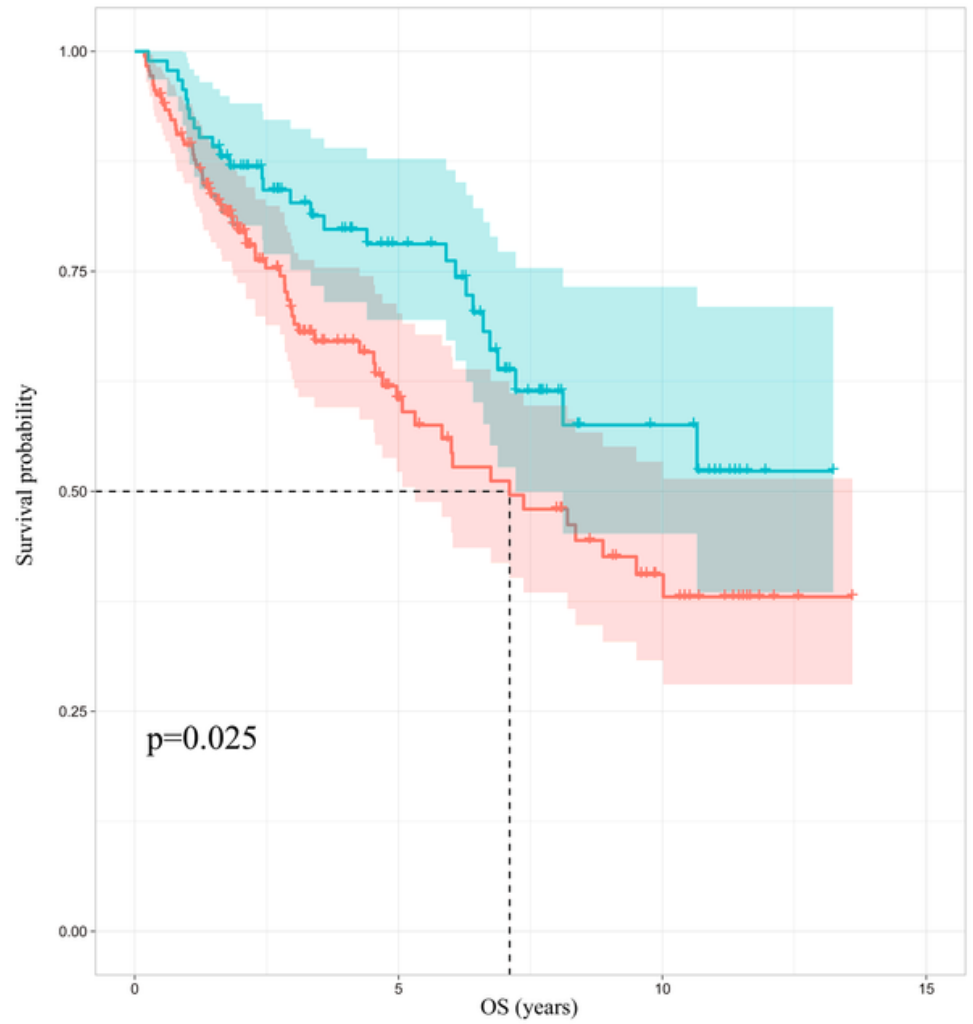

Number at risk

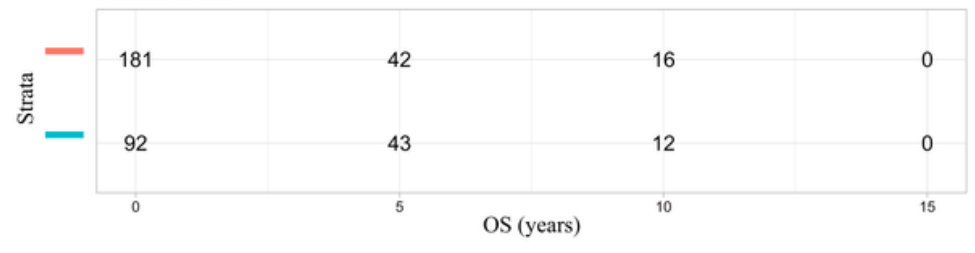

B Strata + No seizures + New-onset seizures

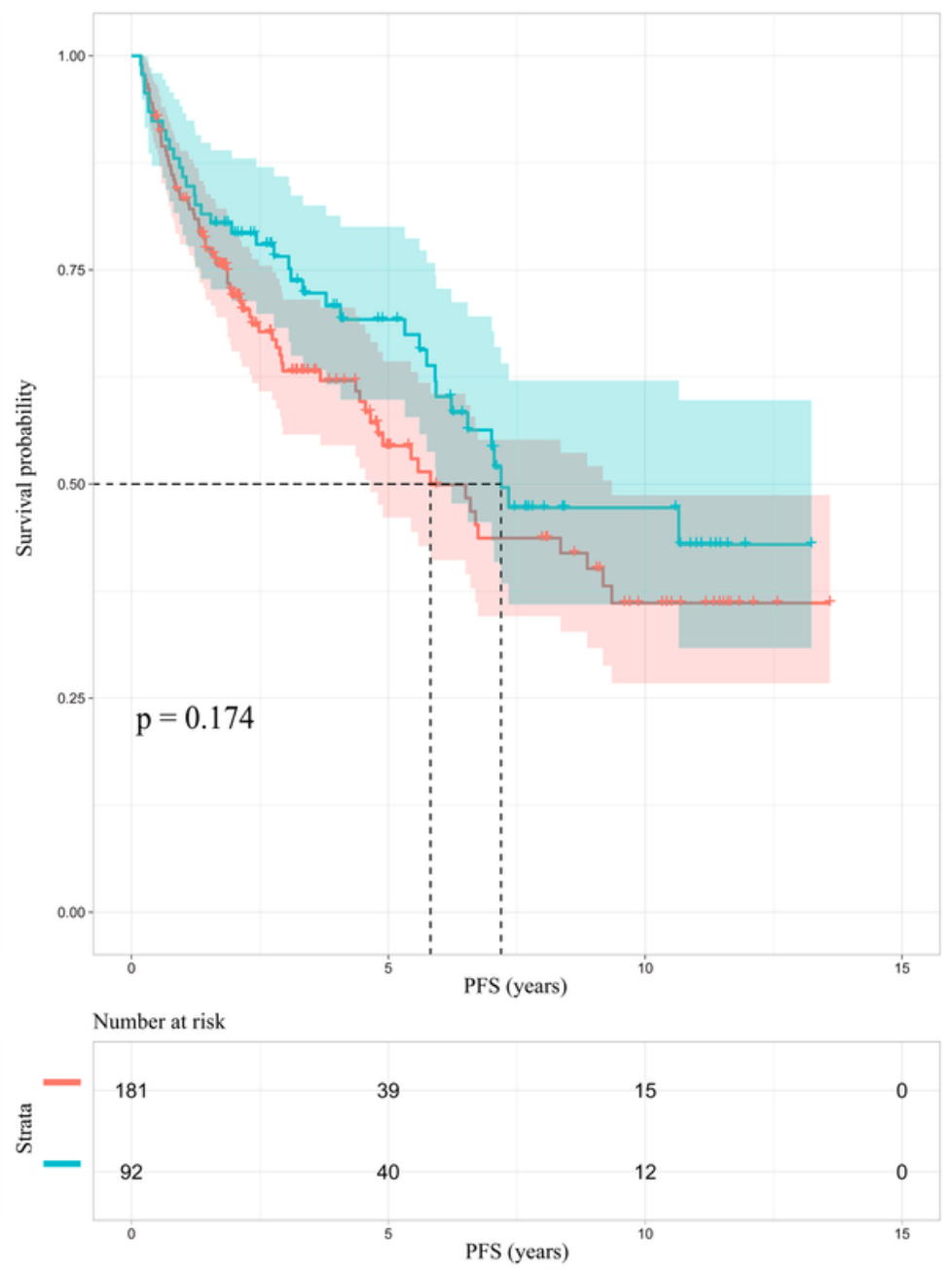

Figure 2

Kaplan-Meier survival analysis in patients without preoperative glioma-related seizures. (A) new-onset postoperative seizures can predict prolonged $O S$ ( $p=0.025$, log-rank test); (B) no significant difference is identified in PFS between patients with new-onset postoperative seizures and those without. OS, overall survival; PFS, progression-free survival. 
Points

Age

Functional deficits

EOR

WHO grade

IDH1 mutation

p53 expression

Ki-67 expression

New-onset seizures

Combined radiochemotherapy

Total Points

1-year survival

5-year survival

10-year survival
2

3

4

$\geq 45$ yrs

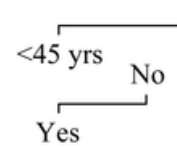

Yes

Non-GTR

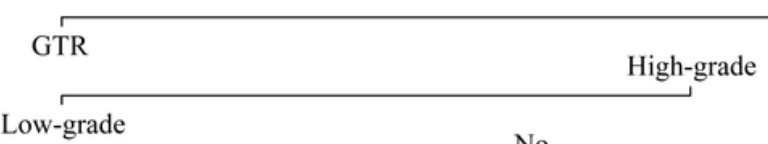

10

9

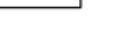

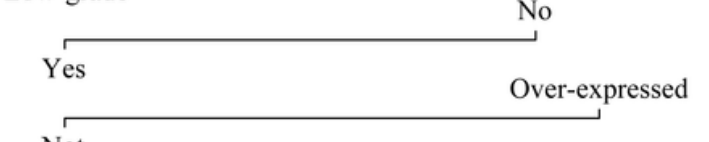

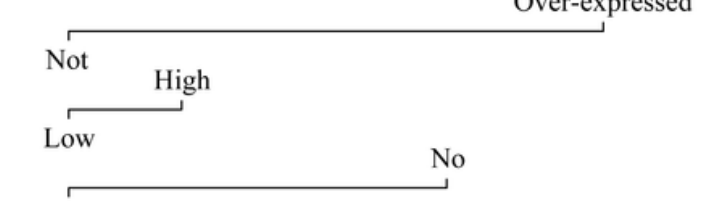

Yes
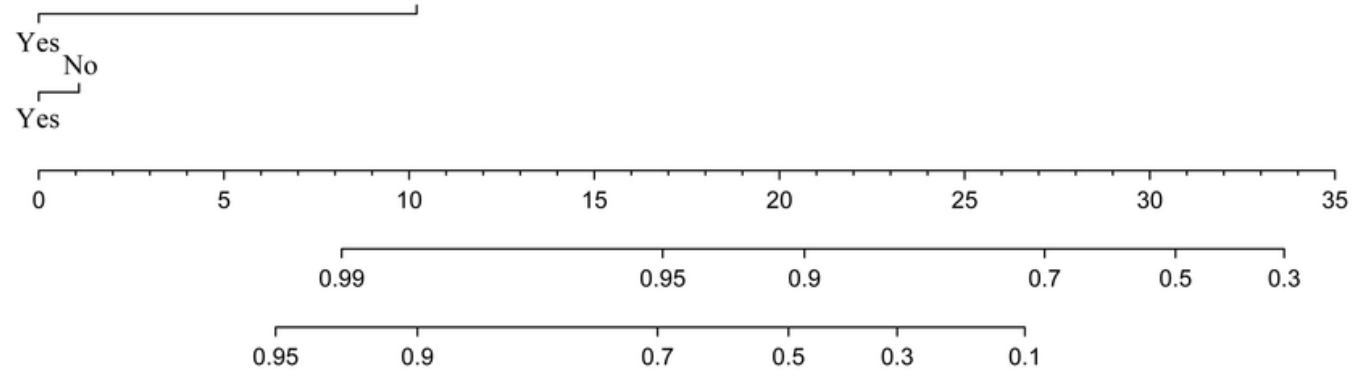

\begin{tabular}{llllll}
\hline 0.95 & 0.9 & 0.7 & 0.5 & 0.3 & 0.1
\end{tabular}

\section{Figure 3}

Prognostic nomogram for survival outcome. The nomogram shows risk factors correlate with the overall survival of patients without preoperative seizures. EOR, extent of resection; WHO, World Health Organization. 